\title{
Um Modelo de Desempenho Multinível para Algoritmos Paralelos Wavefront 2D Clássicos
}

\author{
Alexandre M. Lauredo, Alexandre C. Sena, Maria Clicia S. de Castro, Leandro A. J. \\ Marzulo, Tiago A. O. Alves \\ ${ }^{1}$ Instituto de Matemática e Estatística \\ Universidade do Estado do Rio de Janeiro (UERJ), Rio de Janeiro, Brasil \\ \{alauredo, asena, clicia, leandro, tiago\}@ime.uerj.br
}

\begin{abstract}
The Classic 2D-wavefront pattern is commonly used to parallelize dynamic programming algorithms, where data elements or cells are distributed on a matrix. Since elements in a diagonal are completely independent, they can be computed in parallel. In modern systems, such as multicore clusters, a multi-level parallelism approach is usually adopted and selecting the size of each task is of critical importance to maximize performance. On one hand, coarse-grained tasks will result in low concurrency, while fine-grained tasks increase overhead. In this work we analyse this trade-off, considering aspects such as the task sizes (in two levels) and memory limitations. As a result of this analysis, we propose a model that predicts which task size configurations would yield optimal performance, based on the aforementioned parameters. To evaluate the model accuracy, we use the classic Longest Common Subsequence problem (LCS), which is an important part of DNA sequence alignment.
\end{abstract}

Resumo. O padrão wavefront $2 D$ clássico é comumente usado para paralelizar algoritmos de programação dinâmica, onde elementos ou células de dados são distribuídos em uma matriz. Como os elementos da diagonal são completamente independentes, eles podem ser computados em paralelo. Em sistemas modernos, como os clusters multicore, usualmente é adotada uma abordagem de paralelismo multinível. A seleção do tamanho de cada tarefa tem uma importância crítica para maximizar o desempenho. As tarefas com granularidade grossa resultam em baixa concorrência, enquanto as de granularidade fina podem produzir alta sobrecarga. Neste trabalho analisa-se este balanço (tradeoff), considerando aspectos tais como o tamanho das tarefas (em dois niveis) e limitações de memória. Como resultado desta análise, é proposto um modelo que prediz quais configurações de tamanhos de tarefas produzem alto desempenho, com base nos parâmetros mencionados. Para avaliar a precisão do modelo é usado o algoritmo clássico para encontrar a maior subsequência comum (LCS), que é uma parte importante do alinhamento de sequências de DNA.

\section{Introdução}

O padrão wavefront clássico [Anvik et al. 2002, Mohanty and Cole 2007] é uma das técnicas mais utilizadas para paralelizar algoritmos de programação dinâmica. Uma matriz multidimensional representa a relação de recorrência em algorimos de programação 
dinâmica, onde todos os elementos na mesma diagonal são independentes e podem, portanto, ser calculados em paralelo. No entanto, para diminuir os custos de gerenciamento de thread e de sincronização, os elementos são normalmente organizados em blocos de granularidade grossa que seguem o mesmo padrão de dependência do problema original.

Em clusters multicore modernos, é comum adotar uma implementação híbrida que utiliza a biblioteca MPI para comunicação entre os nós e memória compartilhada dentro de cada nó. Nesta abordagem, é empregada uma matriz wavefront com divisão em dois níveis. A divisão interna é usada para distribuir o trabalho entre os cores, enquanto a divisão externa é usada para distribuir o trabalho entre máquinas do cluster. Neste contexto, uma divisão de dados eficiente é de extrema importância para se conseguir alto desempenho. Além disso, diversas aplicações wavefront são limitadas pela memória. Este fato deve ser observado ao se comprar/escolher um sistema para executar tais aplicações.

Este trabalho propõe um modelo de desempenho multinível para avaliar algoritmos paralelos wavefront, considerando as limitações de memória e custo de paralelização. Como estudo de caso, é implementada uma aplicação que calcula a maior subsequência comum (LCS) entre duas cadeias, sendo este algoritmo importante para aplicações bioinformática. A implementação é baseada na versão MPI descrita em [Chen et al. 2006] e estendida com o paralelismo interno, usando OpenMP e TBB.

Os resultados mostram que o modelo de desempenho pode fornecer um limite superior realístico para o speedup potencial de um sistema específico, considerando a contenção de memória. Além disso, análises de execuções reais mostram que uma estratégia gulosa que inicia com tarefas de granularidade fina e que se tornam mais grossas seguindo o gradiente em cada passo, pode encontrar parâmetros de divisão de tarefa perto dos valores ótimos. Isto não só pode auxiliar os usuários a ajustarem suas aplicações, obtendo bom desempenho em seus sistemas, mas também fornecer a eles um conjunto de diretrizes para especificar um novo sistema ou atualizar um sistema existente.

O restante deste trabalho está organizado da seguinte forma: (i) Seção 2 descreve como é o padrão wavefront $2 D$ clássico e explica o algoritmo LCS; (ii) Seção 3 discute a concorrência no padrão wavefront e apresenta o modelo de desempenho; (iii) Seção 4 descreve a implementação híbrida do LCS; (iv) Seção 5 apresenta os resultados comparando o desempenho previsto pelo modelo com a execução real e discute como a estratégia gulosa poderia ser usada para selecionar os parâmetros de divisão de tarefas; (v) Seção 6 aborda alguns trabalhos relacionados; (vi) Seção 7 conclui e discute os trabalhos futuros.

\section{O Padrão Wavefront 2D Clássico}

O padrão wavefront [Anvik et al. 2002] é amplamente adotado para paralelizar algoritmos de programação dinâmica que podem ser representados por uma relação de recorrência multidimensional. No wavefront é utilizada uma matriz multidimensional, e seus valores são computados de um canto da grade do problema ao canto oposto, atravessando a matriz diagonalmente, como pode ser visto na Figura 1(a). Consequentemente, todos os elementos em uma diagonal são completamente independentes entre si e podem ser computados concorrentemente, permitindo o uso de computação paralela [Mohanty and Cole 2007].

Uma aplicação clássica que emprega o padrão paralelo wavefront é a Longest Common Subsequence (LCS). Dada uma sequência de símbolos $S=\left\langle a_{0}, a_{1}, \ldots, a_{n}\right\rangle$, 


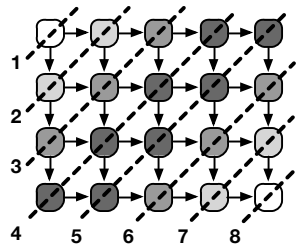

(a) padrão

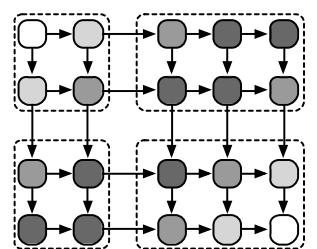

(b) divisão em blocos

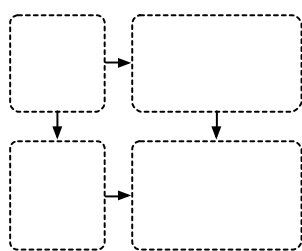

(c) comunicação

Figura 1. Wavefront 2D e divisão em blocos para engrossar o grão.

uma subsequência $S^{\prime}$ de $S$ é obtida, removendo zero ou mais símbolos de $S$. Por exemplo, dado $K=\langle a, b, c, d, e\rangle, K^{\prime}=\langle b, d, e\rangle$ é uma subsequência de $K$. Uma LCS de $X$ e $Y$ é uma subsequência que aparece em $X$ e $Y$ e tem o maior tamanho. O comprimento $M[m, n]$ de uma LCS de sequências $A=\left\langle a_{1}, a_{2}, \ldots, a_{m}\right\rangle$ e $B=\left\langle b_{1}, b_{2}, \ldots, b_{n}\right\rangle$ pode ser definido, recursivamente, como:

$$
M[i, j]= \begin{cases}0 & \text { se } i=0 \vee j=0, \\ M[i-1, j-1]+1 & \text { se } i, j>0 \wedge a_{i}=b_{j}, \\ \max \{M[i-1, j], M[i, j-1]\} & \text { caso contrário. }\end{cases}
$$

Desta definição, proposta em [Wagner and Fischer 1974], pode ser diretamente derivado um algoritmo de programação dinâmica. Além disso, pode ser aplicado o padrão wavefront. Isto porque cada elemento $M[i, j]$ da matriz de valores depende de seus vizinhos superior $(M[i-1, j])$, esquerdo $(M[i, j-1])$ e superior-esquerdo $(M[i-1, j-1])$.

O alinhamento de sequências é um mecanismo extremamente importante para investigar a similaridade entre as espécies. Com Frequência, sequências genéticas semelhantes são relacionadas a grandes similaridades entre estruturas moleculares ou funcionais. Neste contexto, encontrar a maior subsequência comum (LCS) entre duas sequências é uma tarefa essencial no alinhamento de sequências de DNA.

Em razão do enorme crescimento dos bancos de dados de Genomas, como o GenBank, e do tamanho das cadeias de DNA, por exemplo o genoma de um simples Drosophila é composto de 122.653 .977 pares de base, enquanto o genoma humano é formado por $3,3 \times 10^{9}$ pares de base [D. A. Benson and Sayers 2013], algoritmos paralelos são extremamente necessários para comparar grandes sequências. Devido a grande quantidade de memória necessária para comparar grandes cadeias, uma arquitetura de memória distribuída é mais adequada, pois permite a divisão da matriz entre os vários nós do sistema.

Uma implementação multinível do LCS, usando a técnica wavefront, não somente permite executar mais rápidamente, mas também avaliar sequências maiores. Além disso, como o LCS é uma aplicação limitada pela memória, ela se torna uma boa candidata para avaliar o modelo de desempenho proposto.

\section{Análise de Concorrência do Wavefront}

Esta seção analisa brevemente os speedups potenciais que podem ser obtidos em uma implementação paralela wavefront para clusters multicore. Em um cenário real, a criação de uma tarefa para computar somente um elemento da matriz wavefront $(M)$ pode gerar muita sobrecarga. Por isto, uma solução comum é dividir a matriz em blocos, como pode ser visto na Figura 1(b). O padrão de comunicação observado entre os blocos é o mesmo que o do wavefront original, como mostrado na Figura 1(c). Este comportamento fractal 
torna mais fácil para os desenvolvedores escolher o tamanho dos blocos que melhor se ajustam aos seus sistemas, sem se preocupar em reescrever o código. Essa subdivisão de blocos pode ainda ocorrer em múltiplos níveis para se ajustar à arquitetura do sistema.

Em clusters multicore são muito adotadas as implementações híbridas, que usam memória distribuída para comunicação entre nós (MPI) e memória compartilhada (threads) dentro de cada nó. Logo, é necessária uma abordagem em dois níveis composta por paralelismo interno (multicore) e externo (cluster). O speedup potencial interno é limitado pelo número de cores da máquina, enquanto o speedup potencial externo é limitado pelo total de nós. A Figura 2(a) mostra a divisão em dois níveis da matriz de valores para o processamento paralelo em clusters multicore. O primeiro nível é denominado Cluster, que é uma matriz de $C_{H} \times C_{W}$ Blocos, sendo $C_{H}$ e $C_{W}$ a altura e largura do Cluster, respectivamente. Os Blocos são as unidades de trabalho dos processos MPI. No segundo nível, cada Bloco é uma matriz de $B_{H} \times B_{W}$ grãos, sendo $B_{H}$ e $B_{W}$ a altura e largura do Bloco, respectivamente. Finalmente, os grãos são as unidades de trabalho das threads, cada uma representando uma fatia real da matriz de valores a ser computada sequencialmente. A altura e a largura do grão são $G_{H}$ e $G_{W}$, respectivamente.

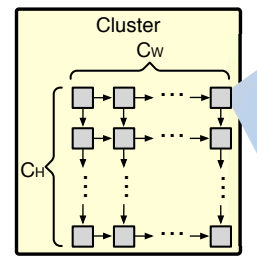

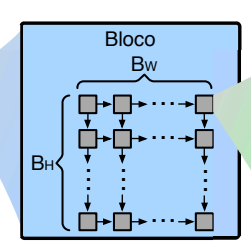

(a) níveis
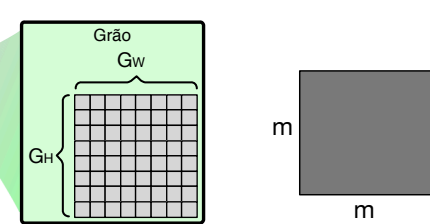

m

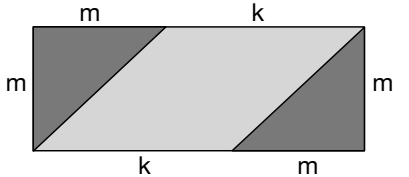

(b) geometria

Figura 2. Divisão em níveis e formato da matriz.

Dada uma matriz wavefront $M$ composta por $m \times n$ elementos, a complexidade do tempo de execução serial é $O(m \times n)$. Por outro lado, o tempo de execução paralelo em um nível é limitado pelo caminho crítico do grafo de dependência wavefront [Arora et al. 1998] (Figura 1(a)). Este caminho vai de $M[0,0]$ a $M[m-1, n-1]$ com comprimento $m+n-1$. Considerando que todos os blocos têm aproximadamente o mesmo tamanho e que o mesmo se aplica aos grãos, todos os caminhos têm o mesmo tempo de execução. Portanto, a Equação 1 representa o Speedup Potencial Interno $\left(S_{I}\right)$, enquanto a Equação 2 representa o Speedup Potencial Externo $\left(S_{E}\right)$.

$$
S_{I}=\frac{B_{H} \times B_{W}}{B_{H}+B_{W}-1}
$$

$$
S_{E}=\frac{C_{H} \times C_{W}}{C_{H}+C_{W}-1}
$$

Para calcular o Speedup Potencial $(S)$ é necessário considerar o número de nós $N$ (máquinas em um cluster) e o número de processadores $P$ (cores na máquina). Isto porque, eles limitam o speedup potencial máximo que pode ser atingido em cada nível. Assim, o speedup potencial é um produto dos Speedups Potenciais Interno e Externo, considerando os limites mencionados anteriormente, como pode ser visto na Equação 3. É importante destacar que o Speedup Potencial máximo é um limite superior quando não são considerados os custos de comunicação para o Speedup Externo e nem os custos de 
gerenciamento e sincronização para o Speedup Interno.

$$
S=\min \left\{\min \left\{S_{I}, P\right\} \times S_{E}, P \times N\right\}
$$

O Speedup Potencial máximo que pode ser obtido em um nível depende da geometria da matriz wavefront. Por exemplo, considere duas matrizes wavefront $M$ e $M^{\prime}$ ambas com 16 elementos, mas com dimensões $4 \times 4$ e $2 \times 8$, respectivamente. Ambas matrizes podem ser vistas como quadriláteros com a mesma área (16), que pode ser relacionada ao tempo de execução sequencial. Por outro lado, o tempo paralelo (tempo do caminho crítico) é diferente ( 7 para $M$ e 9 para $M^{\prime}$ ), podendo ser relacionado com o perímetro (ele é na verdade metade do perímetro). Portanto, o Speedup Potencial de $M(\approx 2.29)$ é melhor que o Speedup Potencial de $M^{\prime}(\approx 1.78)$. De fato, dadas duas matrizes com o mesmo número de elementos (quadriláteros com a mesma área) o Speedup Potencial máximo é atingido em uma matriz quadrada (que tem o menor perímetro). Isto também é conhecido como o Problema Isoperimétrico, apresentado e provado em [Eggleston 1967].

Por outro lado, dadas duas matrizes com a mesma altura e larguras diferentes (áreas diferentes), o melhor Speedup Potencial (relação entre área e perímetro) é atingido pela matriz mais larga. A Figura 2(b) mostra uma matriz quadrada e uma retangular, com a mesma altura $(m)$ e larguras diferentes ( $m$ e $m+k$, respectivamente). A Equação 4 mostra que, neste cenário, a matriz retangular fornece o melhor Speedup Potencial que a matriz quadrada. O lado esquerdo da equação representa o Speedup Potencial para a matriz quadrada, enquanto o lado direito mostra o Speedup Potencial para a matriz retangular.

$$
\frac{m^{2}}{2 \times m-1}<\frac{m^{2}+k \times m}{(2 \times m+k-1},(m \geq 1 \wedge k \geq 1)
$$

Uma razão para fixar a altura da matriz wavefront é quando se deseja executar a aplicação em um cluster e evitar o overhead de comunicação, criando um processo por máquina e dando uma linha de blocos para cada um daqueles processos (como pode ser visto na Seção 4). Neste caso, para aumentar o Speedup Potencial seria necessário ter mais blocos na horizontal (aumentar $C_{W}$ ), resultando numa matriz retangular, como mostrado na Figura 2(b). Como uma consequência, a largura do bloco $\left(B_{W}\right)$ se tornaria menor, incorrendo em mais comunicação entre blocos, de acordo com o padrão wavefront.

A diferença de desempenho entre a memória e o processador pode ter um grande impacto na execução, especialmente quando considera as limitações de memória das aplicações [Sena et al. 2011]. Assim, um speedup teórico que não considera os custos de acesso à memória produz valores não realistas. Ao analisar como o gargalo da memória pode afetar o desempenho de uma aplicação wavefront é possível fornecer um cálculo aproximado dos custos dos acessos a memória e adicioná-los a equação do Speedup Potencial. Diferentemente do speedup teórico (Equação 3), limitações de memória são altamente dependentes das características da aplicação e da máquina.

Primeiro, assuma que a memória principal é o gargalo e que os dados em cache não causam contenção. Com isto em mente, é necessário encontrar quantas instruções são necessárias para computar um elemento na matriz wavefront e qual porção destas instruções acessam a memória principal. Existem pelo menos dois acessos por elemento, 
uma leitura (falha compulsória) e uma escrita (write back). Mesmo se houver mais leituras nesta matriz, a localidade dos dados evita acessos extras a memória principal. Se são utilizadas estruturas de dados auxiliares além da matriz wavefront, deve-se verificar se elas adicionam acessos significativos a memória principal.

Considere que uma thread não pode realizar acessos a memória simultâneos no mesmo core e assuma que o número de instruções por ciclo (IPC) da aplicação é igual a 1 (em um superescalar). A Equação 5 mostra o número máximo de operações de memória (bottleneck da memória) que podem ser realizados simultaneamente se a aplicação é composta por $100 \%$ de acessos a memória $\left(M_{b}\right)$, onde $M_{t}$ é a taxa de transferência da memóra (em MT/s), $M_{c}$ é o número de canais no barramento da memória e $P_{f}$ é a frequência do processador (em MHz). Note que se IPC é diferente de um, é necessário dividir $M_{b}$ pelo IPC. Neste trabalho assume-se que o IPC é igual a um e isto é válido para determinar o limite superior para nosso estudo de caso.

A Equação 6 mostra a razão $\left(M_{\text {ratio }}\right)$ entre o número de instruções de acesso à memória $\left(M_{i}\right)$ e o total de instruções $\left(T_{i}\right)$ por elemento da matriz wavefront. Essa informação pode ser obtida através de ferramentas de profiling, como a Intel $§ V T u n e$ ${ }^{\mathrm{TM}}$ ou Valgrind. Finalmente, a Equação 7 mostra o Speedup Interno máximo $\left(S_{\text {Imax }}\right)$ que pode ser atingido, considerando o gargalo da memória da aplicação.

$$
M_{b}=\frac{M_{t} \times M_{c}}{P_{f}} \quad \text { (5) } \quad M_{\text {ratio }}=\frac{M_{i}}{T_{i}} \quad \text { (6) } \quad S_{\text {Imax }}=\frac{M_{b}}{M_{\text {ratio }}}
$$

Com essa informação, é necessário atualizar o Speedup Potencial (Equação 3) para incluir $S_{\text {Imax }}$. A Equação 8 mostra que se $S_{\text {Imax }}$ é menor que o número real de cores do processador $(P)$ e o Speedup Interno $\left(S_{I}\right)$, ele limita o Speedup Potencial.

$$
S=\min \left\{\min \left\{S_{I}, P, S_{\text {Imax }}\right\} \times S_{E}, P \times N\right\}
$$

\section{LCS Paralelo Híbrido para Clusters Multicore}

Esta seção descreve duas implementações híbridas paralelas do algoritmo LCS que utilizam MPI para comunicação entre os nós e OpenMP ou Intel@Thread Building Blocks (TBB) para comunicação dentro de cada nó. Estas implementações são utilizadas como estudo de caso para analisar o modelo de desempenho proposto.

A implementação MPI é baseada em [Chen et al. 2006] e utiliza comunicação assíncrona somente de baixo para cima. Os processos MPI são organizados em um vetor, onde cada um é responsável por computar uma linha de blocos da matriz. A divisão vertical da matriz em blocos é apenas virtual, uma vez que não há necessidade de comunicação MPI na direção horizontal, dado que a linha inteira de blocos é computada pelo mesmo processo. Cada bloco libera a computação do vizinho de baixo (através de uma mensagem MPI) e do vizinho da direita (sem a necessidade de enviar mensagem), como pode ser visto na Figura 3. A largura do bloco virtual $\left(B_{W}\right)$ é passada como parâmetro.

O trabalho realizado por cada processo MPI pode ser também paralelizado, utilizando memória compartilhada. Dessa maneira, somente um processo MPI por máquina é usado, enquanto múltiplas threads são empregadas. A ideia é dividir o bloco MPI em uma matriz de grãos que possam ser processados utilizando OpenMP ou TBB. No OpenMP, a computação de um bloco é realizada com dois laços aninhados. O laço de fora percorre 
as diagonais da matriz de grãos sequencialmente, enquanto o laço de dentro computa os grãos da diagonal em paralelo, como pode ser visto na Figura 1(a). Por outro lado, no TBB, o conceito de Flow Graph é usado para implementar o padrão wavefront. Nas duas versões, as dimensões do grão $\left(G_{W}\right.$ e $\left.G_{H}\right)$ são iguais e passadas como parâmetros.

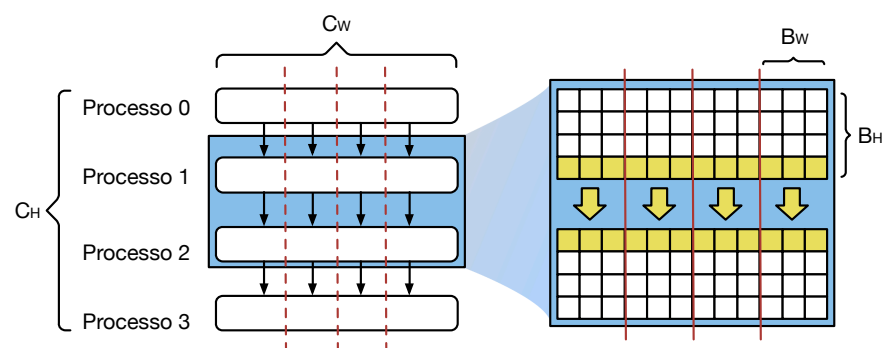

Figura 3. Solução paralela para memória distribuída.

Foram implementadas duas aplicações, usando os conceitos mencionados: OMP+MPI que utiliza uma abordagem de memória distribuída com comunicação assíncrona combinada com OpenMP para o paralelismo dentro de cada nó; TBB+MPI que utiliza uma abordagem de memória distribuída com comunicação assíncrona combinada com TBB para o paralelismo dentro de cada nó.

\section{Análise Experimental}

Esta seção apresenta uma série de experimentos para avaliar o desempenho do algoritmo LCS wavefront variando a granularidade das tarefas, considerando aspectos como o gargalo de memória e sobrecarga na utilização da memória distribuída. Inicialmente é realizado um profiling da aplicação LCS para que se estime a utilização da memória. Em seguida, é apresentada uma análise dos speedups teóricos considerando a configuração de um cluster real. Além disso, é realizada uma avaliação dos custos de usar memória compartilhada em uma máquina real e como isto impacta no speedup potencial. Por último, são apresentados os speedups reais para cada uma das versões híbridas apresentadas, seguida de uma discussão sobre como escolher as melhores configurações de bloco e grão.

Todos os experimentos foram realizados em um cluster composto de 10 nós, onde cada máquina possui dois processadores Intel $₫$ Xeon-E5345, com 4 cores cada um, velocidade de 2,33 GHz, 8GB DDR2 667MT/s com dois canais, interconectados através de uma rede ethernet Gigabit. Nos experimentos foram considerados cenários com 10 processos MPI, um por máquina, e 4 e 8 threads.

Com base nas análises apresentadas na Seção 3, mapas de calor dos spedups potencias (teóricos) (Equação 3) são apresentados nas Figuras 4(a) e 4(b), considerando um cluster composto de 10 nós com 8 e 4 cores, respectivamente. A coordenada $x$ mostra o tamanho de $G_{W}$, enquanto que a coordenada $y$ o tamanho de $B_{W}$.

Os speedups teóricos aumentam à medida que a granularidade das tarefas diminui, o que significa menores valores para $G_{W}$ e $B_{W}$. Assim, o melhor speedup teórico é alcançado na primeira célula do mapa de calor $(500 \times 25)$. É importante destacar que $G_{W}$ define a granularidade das tarefas do nível mais interno (grão), enquanto que $B_{W}$ define a granularidade das tarefas do nível externo (bloco). 


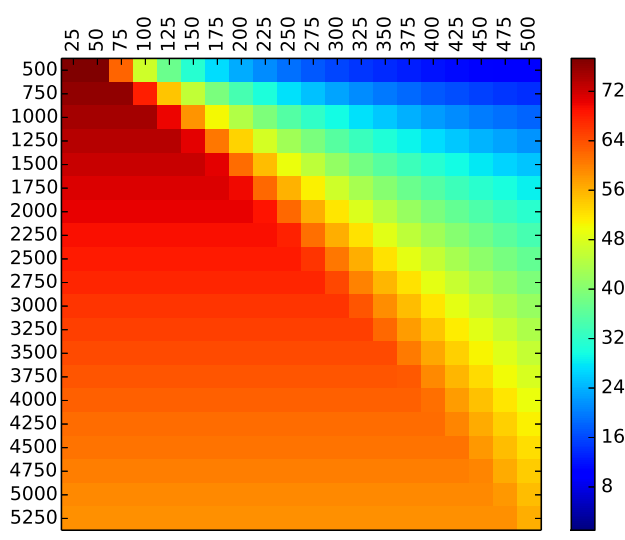

(a) 10 nós com 8 cores

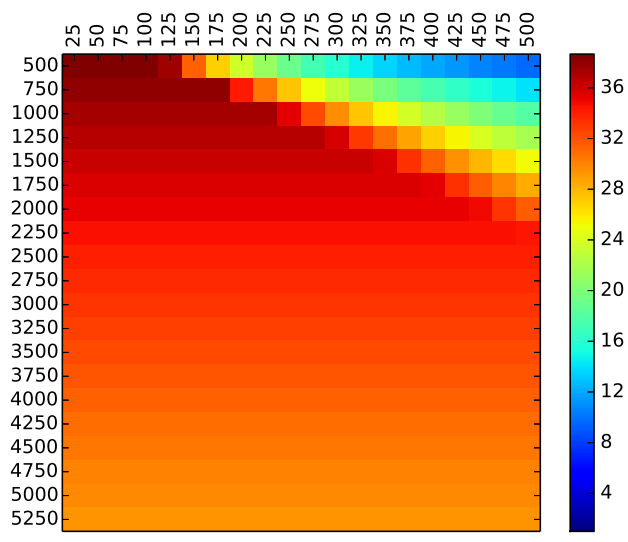

(b) 10 nós com 4 cores

Figura 4. Speedups potenciais (teóricos).

Embora os mapas de calor com 4 e 8 cores sejam diferentes, o comportamento é similar, o que significa que o speedup aumenta a medida que se move para o lado esquerdo superior da matriz. Entretanto, o speedup teórico com 8 cores é, em geral, duas vezes melhor do que com 4 cores, o que era esperado, uma vez que os speedup internos teóricos (Equação 1) resultaram exatamente na quantidade de cores, 8 e 4, respectivamente.

Portanto, para um cluster sem limitações no acesso a memória e nenhum custo de paralelização, o melhor seria escolher os menores valores possíveis para $G_{W}$ e $B_{W}$. Entretanto, como tal cenário não existe, a seguir são analisados o impacto de tais custos e limitações no speedup teórico e, mais importante, a escolha de $G_{W}$ e $B_{W}$ que maximize o speedup.

Para avaliar o gargalo de memória, o Valgrind foi usado para mensurar a aplicação LCS e os resultados mostraram que 11 instruções são necessárias por elemento, onde 5 das quais são de memória, mas somente 2 acessam a memória principal, como discutido na Seção 3. Assim, LCS tem $M_{\text {ratio }}=0.1818$ e $M_{b}=0.5718$, resultando em $S_{\text {Imax }}=$ 3.1452. Isto significa que os 8 cores disponíveis não serão eficientemente utilizados, como mostra a Figura 5(a), que é igual para 8 e 4 cores. A razão para este comportamento é simples: embora os nós com mais cores forneçam melhores speedups teóricos, na prática os acessos à memória limitam o speedup interno. Além disso, embora o comportamento deste novo speedup teórico seja o mesmo observado na Figura 4, os valores do speedup reduziram para menos da metade dos valores originais, no cenário com 8 cores.

Estes resultados são de extrema importância na hora de se comprar (ou escolher) um sistema com a melhor relação entre custo/desempenho para uma aplicação wavefront. A Figura 5(b) apresenta os valores de $S_{\text {Imax }}$ para processadores com diferentes frequências e taxas de transferência de memória, em sistemas com 2 canais de acesso à memória. Uma boa opção para maximizar o desempenho é escolher a frequência do computador e quantidade de cores após calcular $S_{\text {Imax }}$, que será maior caso sejam escolhidas memórias com altas taxas de transferência e placas mãe com maior número de canais.

Embora o gargalo de memória limite o speedup teórico, o comportamento do mapa de calor é o mesmo, ou seja, o speedup aumenta à medida que os valores $G_{W}$ e $B_{W}$ diminuem (granularidade fina). Entretanto, em um cenário real, o aumento de tarefas de 
granularidade cada vez mais fina, provavelmente, aumentará a sobrecarga. A seguir, é analisado o impacto do custo de gerenciamento com o aumento no número de tarefas. Para medir a sobrecarga do gerenciamento de tarefas em um ambiente de memória compartilhada, uma aplicação com padrão wavefront foi executada, contendo apenas os laços e diretivas do OpenMP. Esta aplicação é basicamente um esqueleto do padrão wavefront.

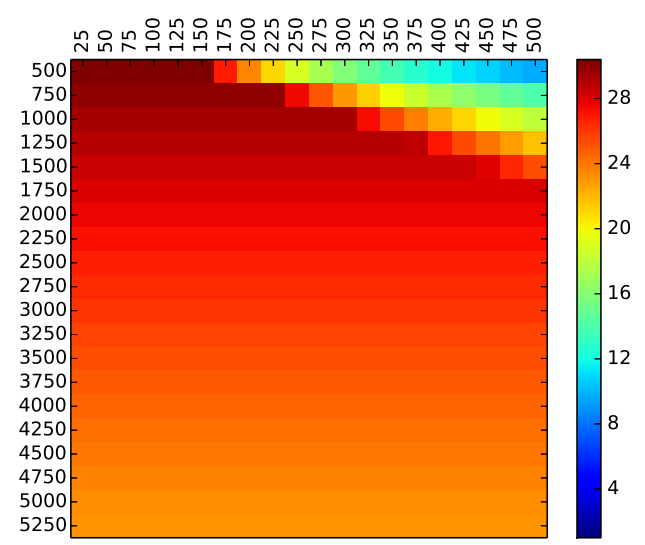

(a) 10 nós com 8 cores e 10 nós com 4 cores

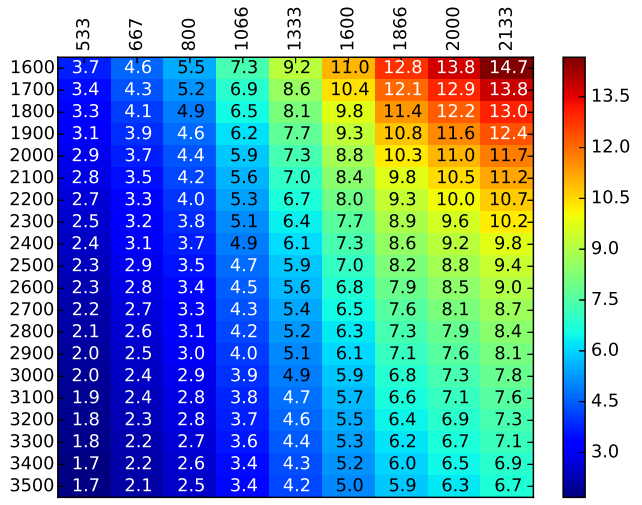

(b) processador $\times$ memória em uma máquina

Figura 5. Speedups teóricos com limitações de memória

O tempo de execução dessa aplicação (em ms), variando os valores de $G_{W}$ e $B_{W}$, pode ser visto na Figura 6 (a). No pior caso a sobrecarga foi $\approx 0,5 \%$ do tempo de execução da aplicação. Entretanto, o gerenciamento de tarefas do OpenMP inclui um número significativo de operações de memória, como apresentado na Figura 6(b). Estas operações de memória são realizadas em estruturas de dados pequenas que provavelmente permanecem na cache durante toda execução do programa e, em aplicações reais, elas podem aumentar a contenção nos acesso a cache, piorando o tempo de execução.

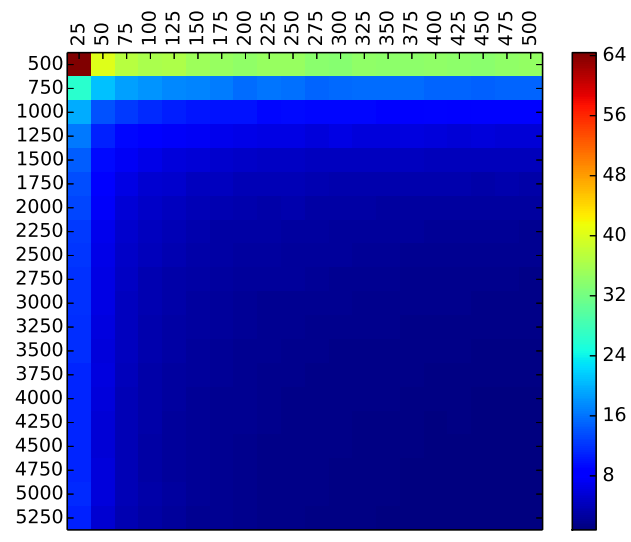

(a) Tempo (ms)

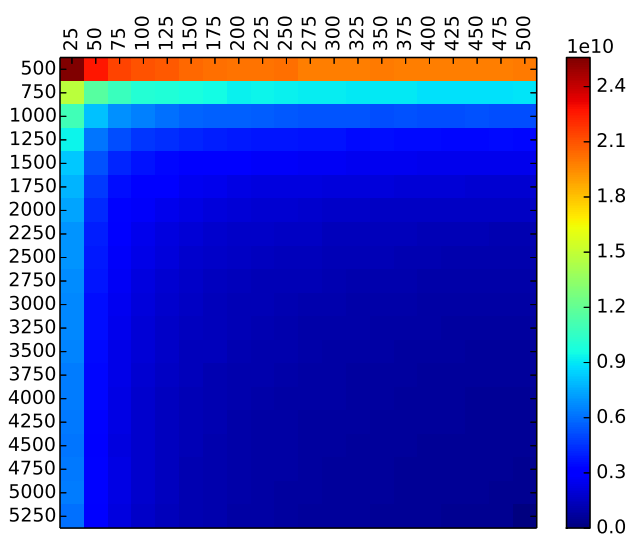

(b) Operações de Memória

Figura 6. Custos do OpenMP.

Como esperado, contrariamente ao comportamento de todos os outros mapas de calor, a medida que os valores de $G_{W}$ e $B_{W}$ diminuem o tempo de execução cresce, aumentando a sobrecarga (Figura 6(a)). Este mesmo comportamento foi observado na 
quantidade de operações de memória, onde o maior número de operações de memória foi obtido com $G_{W}=25$ e $B_{W}=500$. Este experimento claramente mostra o efeito colateral de aumentar-se a quantidade de tarefas de granularidade fina.

Finalmente, a Figura 7 apresenta mapas de calor com speedups reais para as aplicações LCS OMP+MPI e TBB+MPI, em 10 nós com 4 e 8 cores, ao alinhar cadeias de DNA compostas de $130 k$ pares de base. Cada cenário, variando os valores de $G_{W}$ e $B_{W}$, foi executado 20 vezes e o tempo médio foi utilizado para calcular o speedup. O desvio padrão foi calculado demonstrando valores desprezíveis. Repare que as escalas para 8 e 4 cores são diferentes para melhorar a visualização do comportamento dos speedups. Para as duas versões híbridas, foram usados 10 processos MPI (1 por máquina), enquanto que a quantidade de threads por nó é igual a 4 (Figuras 7(a) e 7(c)) e 8 (Figuras 7(b) e 7(d)).

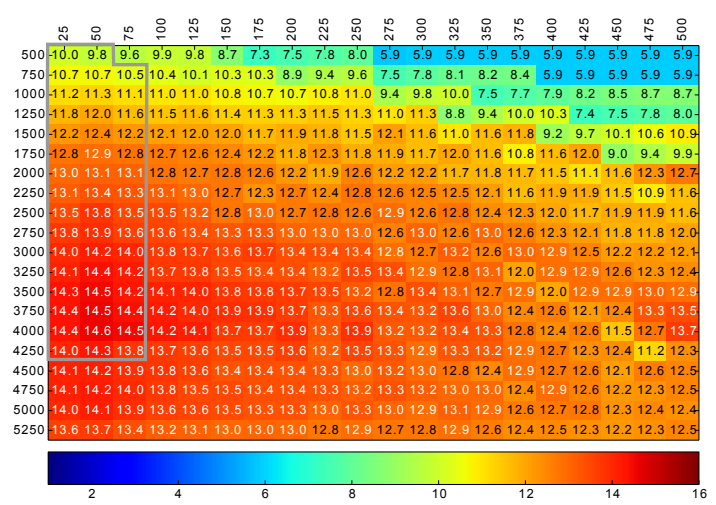

(a) 4 cores - OMP+MPI

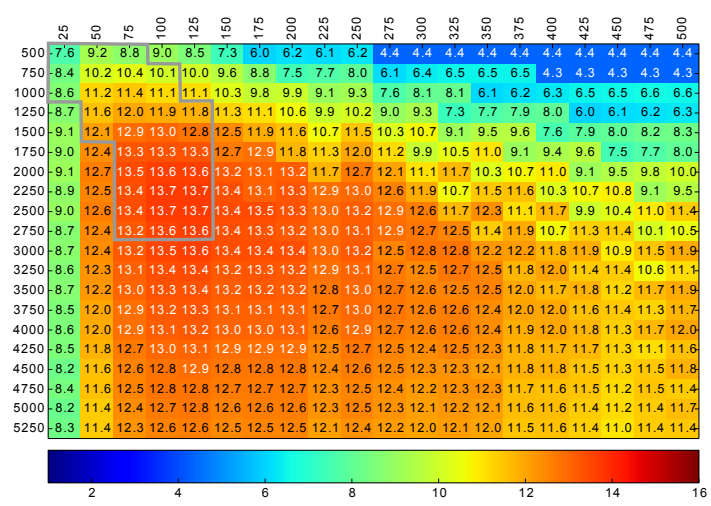

(c) 4 cores - TBB+MPI

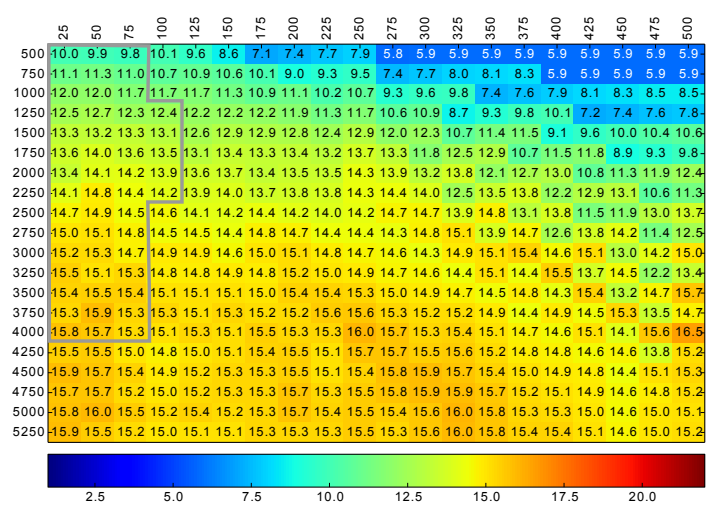

(b) 8 cores - OMP+MPI

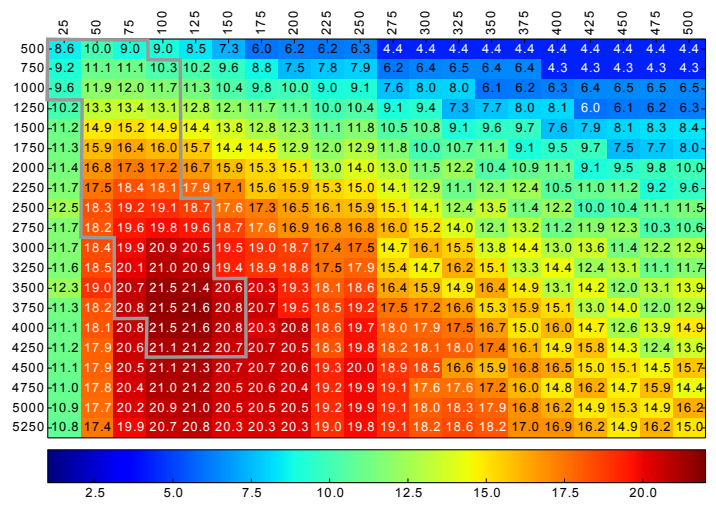

(d) 8 cores - TBB+MPI

Figura 7. Speedups para LCS híbrido.

Diferentemente de todos os outros mapas de calor, os melhores speedups estão localizados no centro esquerdo do mapa de calor, ao invés de estar localizado na parte superior esquerda. Este comportamento representa o balanceamento entre o paralelismo potencial e o custo de gerenciamento. Além disso, enquanto o limite superior representado pelo speedup teórico com contenção de memória (Equação 8) foi 28, o speedup máximo para as versões MPI+OMP e MPI+TBB foram 16, 5 e 21, 6, respectivamente. Os maiores custos de gerenciamento e, principalmente, a sincronização do OpenMP prejudicaram bastante o desempenho, enquanto a habilidade de descrever a aplicação como um flow graph do TBB, diminuiu as barreiras, permitindo um desempenho melhor. 
Os Speedups teóricos que consideram o gargalo de memória sugerem que utilizar mais do que 4 cores não aumenta o desempenho $\left(S_{\text {Imax }}=3.1452\right)$. Isto somente acontece se a implementação paralela estiver aproveitando o desempenho máximo do sistema ou se ela mantiver a pressão no barramento de memória no mesmo nível do que a versão sequencial. No caso da versão MPI+OMP esta suposição é verdadeira, visto que nenhuma melhora significativa foi alcançada na execução com 8 cores (figuras 7(a) e 7(b)). Por outro lado, na versão MPI+TBB foram observadas melhoras significativas utilizando 8 cores (figura 7(c) e 7(d)). Isto sugere que os custos do TBB não aumentam a pressão no barramento, podendo inclusive aumentar a quantidade de instruções que não acessam a memória, diminuindo $M_{\text {ratio }}$ e aumentando $S_{I \max }$ (Equações 6 e 7).

Outro aspecto interessante do mapa de calor das aplicações híbridas LCS é mostrar que várias configurações de $G_{W}$ e $B_{W}$ resultaram em valores de speedups próximos do ótimo e, mais ainda, relativamente perto uns dos outros. Assim, pode ser possível encontrar configurações de bloco e grão próximas do valor ótimo sem a necessidade de executar todas as 400 combinações. Assumindo que a especificação de todos os custos de gerenciamento, comunicação e de contenção da memória é dependente da arquitetura, como analisado neste trabalho, uma estratégia gulosa pode ser usada para achar uma boa configuração. Começando com os menores tamanhos de grão e bloco (25 e 500) e iterativamente testando os vizinhos, seguindo o gradiente a cada passo. Na Figura 7, o caminho da estratégia gulosa em cada cenário é destacado com uma linha cinza sólida. A melhor solução foi encontrada em três dos quatro cenários. No caso da versão MPI+OMP com 8 cores um ótimo local foi encontrado, sendo apenas $4 \%$ pior do que a melhor solução.

\section{Trabalhos Relacionados}

Um framework baseado em aprendizagem de máquina para aplicações wavefront foi apresentado em [Mohanty and Cole 2007]. Ele facilita o particionamento dos dados em sistemas compostos de CPUs multicore com múltiplos aceleradores GPU. Não são apresentadas análises do comportamento da aplicação wavefront.

A perda de desempenho na execução de matrizes 3D em sistemas multicore foi observada em [Sena et al. 2011]. Os autores, através da avaliação de desempenho da aplicação RTM usando a ferramenta Intel VTune, detectaram que a principal razão para não se obter speedup linear dentro das máquinas multicore foi o gargalo da memória. Embora, as técnicas de tiling melhorem os acessos a memória, a diferença de velocidade entre memória e processador prejudica o barramento da memória e aumenta as falhas da cache, ocorrendo perda de desempenho, especialmente quando todos os cores são usados.

Uma implementação MPI do algorimo LCS baseada em Needleman-Wunsch foi apresentada em [Chen et al. 2006] que, para evitar as comunicações horizontais, divide a matriz em colunas virtuais de blocos e cada processador é responsável por computar uma ou mais linhas de blocos. Entretanto, os experimentos foram executados em um cluster de máquinas com um único core com somente $256 \mathrm{MB}$ de memória cada, limitando o tamanho das sequências. Além disso, este trabalho não fornece análise de desempenho teórica e não avalia a relação entre tamanho de blocos e desempenho.

\section{Conclusões e Trabalhos Futuros}

Wavefront é uma técnica importante para paralelizar algoritmos de programação dinâmica. 
Entretanto, encontrar uma divisão de dados para executar em clusters multicore não é uma tarefa fácil, que depende não somente do padrão de paralelismo, mas também da arquitetura alvo e dos custos de paralelização.

Este trabalho propôs e analisou um modelo de desempenho multinível para aplicações wavefront. Os resultados mostraram que ele fornece limites superiores aproximados do speedup, considerando a contenção de memória. Mais importante, ele pode não só ser usado para ajudar escolher ou comprar um cluster mais adequado para a aplicação alvo, mas principalmente, avaliar se o desempenho da aplicação wavefront está adequado. Além disso, uma estratégia gulosa pode ser usada para descobrir divisões de dados próximas do ótimo, permitindo uma execução eficiente.

Como trabalhos futuros, para entender melhor as implicações do modelo proposto, incluindo as análises dos custos de paralelização e também o gargalo de memória, será necessário executar o estudo de caso LCS em diferentes sistemas de computadores que permitam avaliar, por exemplo, quão próximo de $S_{\text {imax }}$ é possível chegar. Tais sistemas devem ter uma quantidade grande de cores, memórias mais rápidas e com mais canais. Outro ponto importante a ser avaliado é a influência do tamanho da cache no modelo.

\section{Agradecimentos}

À FAPERJ (processo E-26/203.537/2015), ao CNPq, à CAPES e ao NCC/UNESP pelo apoio aos autores deste trabalho.

\section{Referências}

Anvik, J., MacDonald, S., Szafron, D., Schaeffer, J., Bromling, S., and Tan, K. (2002). Generating parallel programs from the wavefront design pattern. In Proceedings International Parallel and Distributed Processing Symposium., IPDPS 2002, pages 8 pp-.

Arora, N. S., Blumofe, R. D., and Plaxton, C. G. (1998). Thread scheduling for multiprogrammed multiprocessors. In Proceedings of the Tenth Annual ACM Symposium on Parallel Algorithms and Architectures, pages 119-129, New York, NY, USA. ACM.

Chen, Y., Yu, S., and Leng, M. (2006). Parallel sequence alignment algorithm for clustering system. In Shangai. Knowledge Enterprise: Intelligent Strategies in Product Design, Manufacturing, and Management, pages 311-321.

D. A. Benson, M. Cavanaugh, K. C. I. K.-M. D. J. L. J. O. and Sayers, E. W. (2013). Genbank. Nucleic Acids Research, 41:36-42.

Eggleston, H. G. (1967). The isoperimetric problem. In Press, P., editor, Exploring University Mathematics, volume 1, chapter 7. Pergamon Press.

Mohanty, S. and Cole, M. (2007). Autotuning wavefront applications for multicore multigpu hybrid architectures. In Proceedings of Programming Models and Applications on Multicores and Manycores, PMAM'14, pages 1:1-1:9, New York, NY, USA. ACM.

Sena, A. C., Nascimento, A. P., Boeres, C., Rebello, V., and Bulcao, A. (2011). An approach to optimise the execution of $\mathrm{rtm}$ algorithm in multicore machines. In $E$ Science (e-Science), 2011 IEEE 7th International Conference on, pages 403-410.

Wagner, R. A. and Fischer, M. J. (1974). The string-to-string correction problem. J. ACM, 21(1):168-173. 